\title{
«La communication en mutation perpétuelle»
}

\author{
Interview: Bruno Kesseli
}

Dr méd. et lic. phil., rédacteur en chef

Jacqueline Wettstein a travaillé en tant que responsable de la communication aux avant-postes de la FMH. Aujourd'hui, elle prend un nouveau départ professionnel. Dans cette interview, elle livre un court bilan de son activité au service du corps médical depuis 2007, et de son contact soutenu et presque quotidien avec la rédaction du BMS. C'est pour cette raison que nous nous permettons de la tutoyer dans cet entretien.

\begin{abstract}
Tu as contribué à la FMH pendant dix ans en tant que responsable de la communication. Quelles étaient tes attentes lorsque tu as pris ce poste en 2007?

Le domaine de la santé m'était déjà familier grâce à de précédentes activités professionnelles dans une assurance-maladie et dans une agence de relations publiques; je connaissais également le Bulletin des médecins suisses. Par contre, l'association en elle-même, le fonctionnement d'une organisation professionnelle
\end{abstract}

«De mon point de vue, il y a des avantages certains à ce que les responsables de communication aient accès au savoir médical spécialisé sans être eux-mêmes médecins.»

nationale ainsi que le contexte politique étaient nouveaux pour moi. J'ai eu la possibilité, avant de pourvoir ce poste, d'avoir un aperçu de la FMH dans le cadre d'études supérieures et la rédaction d'un mémoire; cela a certainement facilité mon entrée. J'étais impatiente d'effectuer des tâches passionnantes dans un milieu intéressant. Rétrospectivement, je peux affirmer que la FMH a tenu toutes ses promesses.

\section{A-t-il été difficile pour toi de plonger dans la com- plexité du système de santé sans être médecin?}

Au-delà du savoir médical, pour que cette association professionnelle réussisse en politique, dans les médias et les relations publiques, elle doit connaître le droit, l'économie, les statistiques, mais aussi être compétente dans des domaines spécialisés comme l'eHealth ou la santé publique (prévention). Nous, l'équipe chargée de la communication, avions toujours accès à ce

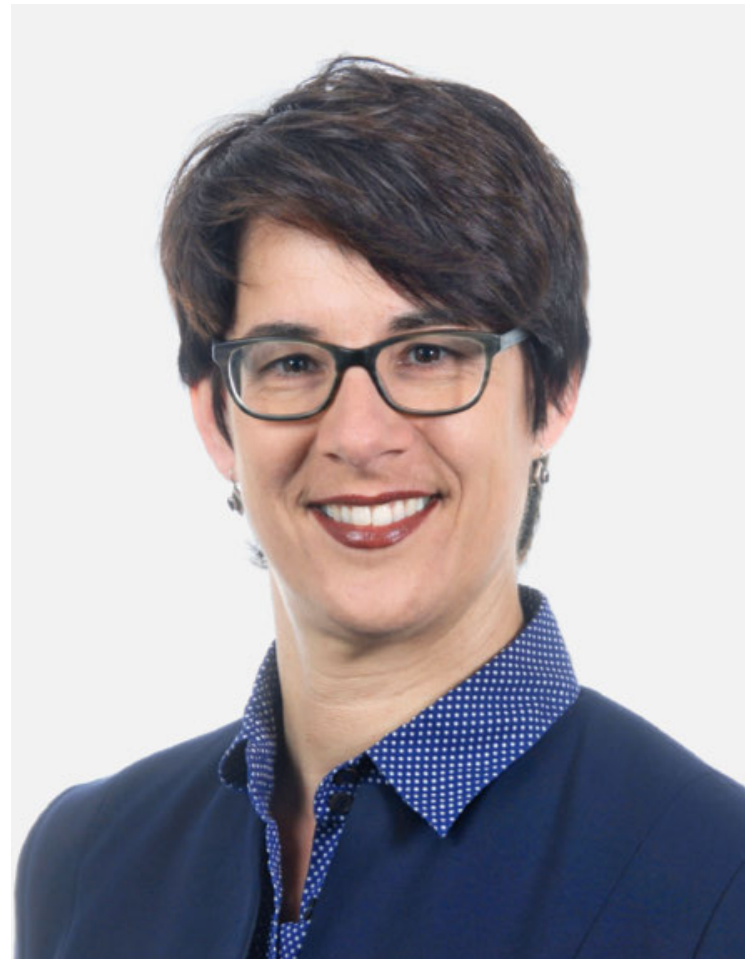

Jacqueline Wettstein quitte la FMH après dix ans d'activité.

savoir par le biais des divisions et services du Secrétariat général de la FMH, des sociétés de discipline ou des organisations faîtières. Une des tâches centrales de la communication est de traduire le savoir interne très pointu en un langage accessible et compréhensible. De mon point de vue, il y a des avantages certains à ce que les responsables de la communication aient accès au savoir médical spécialisé sans être eux-mêmes médecins. 


\section{Comment la communication de la FMH s'est-elle} développée ces dix dernières années?

Je pourrais parler sommairement de deux phases: lors de mes premières années à la FMH, la priorité était avant tout de consolider le travail médiatique et de renforcer la communication, notamment avec les organisations de médecins affiliées. Ensuite, après

«De mon point de vue, le fait que le "bulletin jaune' ne soit pas seulement l'organe officiel de la FMH, mais qu'il reflète aussi la diversité du corps médical et constitue ainsi une plateforme de discussion, est un énorme plus.»

quelques années, nous avons professionnalisé et optimisé les activités dans ce que les Anglo-Saxons appellent le Public Affairs (travail de lobbying) afin de pouvoir participer le plus tôt possible au processus d'élaboration des lois.

\section{Combien de fois as-tu dû expliquer ce que signifie le sigle «FMH»?}

Presque jamais. Même si la dénomination latine n'est pas courante, la population associe très rapidement ces trois lettres aux médecins, à la médecine et à la santé publique, avec une connotation plutôt positive. C'est d'ailleurs ce qu'a démontré un sondage de la FMH auprès de la population en 2014. Il en est ressorti que le sigle «FMH» représentait une marque forte ou un label convainquant. La notoriété - mais aussi la renommée de la FMH contribuent de manière significative à ce que l'opinion de l'association professionnelle des médecins soit sollicitée, notamment par les politiciens, les journalistes, mais aussi le public.

\section{Une question m'intéresse du point de vue du BMS: est-ce que le fait que le BMS soit en même temps un organe officiel de la FMH et une revue ayant une rédaction indépendante a-t-il rendu ta vie profession- nelle difficile?}

Le Bulletin des médecins suisses est une publication établie de longue tradition qui compte parmi ses lecteurs, outre les médecins, des politiciens, des journalistes ainsi que d'autres acteurs de la santé: les journalistes s'informent régulièrement via des thèmes ou des articles du Bulletin des médecins suisses. En tant que responsable de la communication, j'ai beaucoup apprécié la partie FMH du BMS, car elle lui permet de présenter régulièrement ses travaux et son point de vue dans un média largement ancré. De mon point de vue, le fait que le «bulletin jaune» ne soit pas seulement l'organe officiel de la FMH, mais qu'il reflète aussi la diversité du corps médical et qu'il consti- tue ainsi une plateforme de discussion, est un énorme plus.

\section{Quels événements ou aspects de ton travail resteront dans ta mémoire comme des points forts de ta carrière?}

Il y en a plusieurs: par exemple ma première campagne de votation en 2008, mais aussi les ateliers avec les responsables de communication des différentes organisations de médecins. Le dernier gros point fort aura certainement été la conférence de presse de la FMH sur la deuxième intervention tarifaire du Conseil fédéral à laquelle ont assisté de nombreux journalistes et qui a mené à la publication de nombreux articles.

\section{D’après ton expérience, quelles sont les plus grandes forces de la FMH?}

La FMH est, avec plus de 40000 membres, une des plus grandes associations professionnelles nationales en Suisse. Son fort enracinement au sein du corps médical contribue à ce que la FMH compte parmi les acteurs principaux de la santé. L'intérêt médiatique et les invitations à des auditions en sont les témoins. Tout l'art de cette association professionnelle faîtière consiste entre autres à rassembler ses membres hétérogènes ainsi qu'à concilier les diverses opinions des différentes organisations de médecins. A cet égard, je souhaite à la FMH qu'elle puisse à l'avenir s'affirmer encore plus efficacement d'une seule et même voix forte.

\section{A ton avis, sur quoi devrait se focaliser la communica- tion de la FMH dans les prochaines années?}

D'une part, il faut continuer à reconsidérer et optimiser les activités existantes. D’autre part, il est conseillé de renforcer la communication avec les membres et d'axer celle-ci plus fortement sur les besoins des médecins. La communication est en mue constante grâce aux avancées technologiques: en tant que consommateurs, nous voulons être informés en temps réel sur un événement et pouvoir participer. Nous ne désirons plus seulement recevoir les nouvelles sous forme de texte rédigé, mais nous voulons aussi pouvoir les vivre

«Tout l'art de cette association professionnelle faîtière consiste entre autres à rassembler ses membres hétérogènes ainsi que concilier les diverses opinions des différentes organisations de médecins."

en son et en image. Les moyens de communication sont aujourd'hui beaucoup plus nombreux qu'autrefois: la FMH se trouve ainsi devant le choix passionnant d'adapter encore mieux ses contenus et ses ca- 
naux de communication à ses différents partenaires et de cultiver le dialogue avec eux.

\section{Qu'est-ce qui t'attire après la FMH?}

Le domaine de la santé a plein de facettes. Pour cette raison, il était clair pour moi que je voulais encore travailler dans ce secteur. A partir de septembre, je vais diriger le service de communication de l'ipw (Integrierte Psychiatrie Winterthur - Zürcher Unterland). Je me réjouis de me familiariser avec le milieu psychiatrique et le fonctionnement d'une institution qui poursuit depuis plus d'une décennie - et avec succès un système de traitement intégré: l'ipw dispose de services ambulatoires et hospitaliers ainsi que de cliniques de jour répartis sur 15 différents sites. Elle

\section{"Ce qui va manquer est à coup sûr la diversité} culturelle et linguistique que j'ai pu côtoyer à Berne au sein d'une organisation nationale.»

couvre aussi toutes les pathologies psychiatriques rencontrées chez les (jeunes) adultes et les plus vieux. Ce que je trouve intéressant c'est que l'ipw s'engage, en tant qu'employeur, en faveur du temps partiel à tous les niveaux hiérarchiques ainsi que pour la conciliation de la vie familiale et professionnelle. Pour cela, elle est la première clinique psychiatrique de Suisse à avoir obtenu, en 2016, le label «Famille \& Profession». Après la FMH, je me réjouis de ce nouveau sigle de trois lettres, de thèmes passionnants et du travail au cœur de la santé.

\section{Qu'est-ce qui va te manquer? Qu'est-ce qui ne va pas te manquer?}

Ce qui va manquer est à coup sûr la diversité culturelle et linguistique que j'ai pu côtoyer à Berne au sein d'une organisation nationale. Mes collègues du Secrétariat général, mes nombreuses et nombreux autres interlocutrices et interlocuteurs externes et, plus particulièrement, l'équipe de la communication de la FMH vont également me manquer. Ce qui est valable pour la médecine l'est aussi pour la communication: une bonne collaboration entre les disciplines ainsi qu'une équipe bien rodée sont les facteurs déterminants pour une communication réussie. Ce qui ne me manquera pas sont les longs trajets de Zurich à Berne, même si je m'y suis faite au fil des années et que je sais comment mettre ce temps à profit.

\section{Votre communication est entre}

\section{de bonnes mains}

La division Communication de la FMH est à la disposition des membres, des journalistes et des acteurs de la santé pour toutes les questions et les sujets concernant les médecins. La suppléante de la division Communication, Cornelia Steck, assume la direction par intérim jusqu'à l'arrivée de la remplaçante ou du remplaçant de Mme Wettstein. kommunikation[at]fmh.ch, 0313591153 